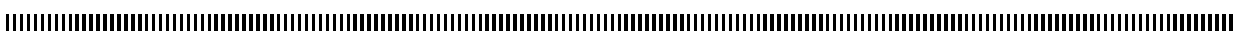

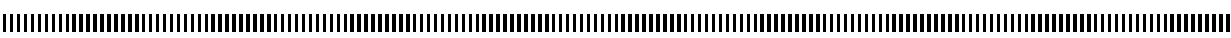
| |

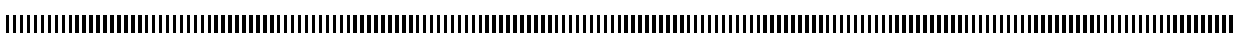
| | |

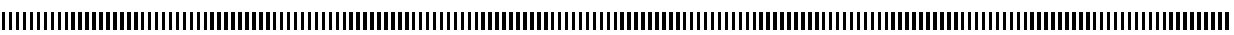
|

Special issue CARl'12

\title{
An arbitrary high order discontinuous Galerkin scheme for the elastodynamic equations
}

\author{
Serge MOTO MPONG \\ University of Yaoundé 1, Cameroon \\ serge_moto@yahoo.com
}

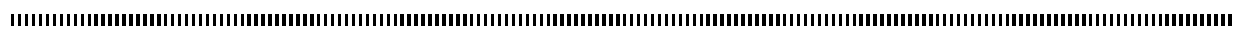

ABSTRACT. We present in this paper the formulation of a non-dissipative arbitrary high order time domain scheme for the elastodynamic equations. Our approach combines the use of an arbitrary high order discontinuous Galerkin interpolation with centred flux in space, with an arbitrary high order leapfrog scheme in time. Numerical two dimensionnal results are presented for the schemes from order two to order four. In these simulations, we discuss of the numerical stability and the numerical convergence of the schemes on the homogeneous eigenmode problem. We also show the ability of the computed schemes to carry out more complex propagation probems by simulating the Garvin test with an explosive source. The results show the high accuracy of the method, both on triangular regular and irregular meshes.

RÉSUMÉ. Nous présentons dans ce papier une méthode Galerkin discontinu d'ordre arbitrairement élevé pour les équations de l'élastodynamique en domaine temporel. Notre approche combine une interpolation spatiale d'ordre arbitraire, des flux centrés ainsi qu'un schéma saute-mouton d'ordre arbitrairement élevé pour l'intégration temporelle. Des résultats numériques de la propagation d'un mode propre 2D sont présentés dans le cas des schémas saute-mouton d'ordre 2 et 4 . Une étude numérique de la stabilité et de la convergence de la méthode est également proposée, ainsi qu'une application du schéma à la résolution d'un problème de propagation plus complexe: le test de Garvin. Ces différentes simulations montrent que le schéma développé est très précis, aussi bien sur des maillages réguliers que non réguliers.

KEYWORDS : elastodynamic equation, discontinuous Galerkin method, leapfrog scheme, centered scheme

MOTS-CLÉS : équation élastodynamique, méthode Galerkin discontinu, schéma saute-mouton, flux centré

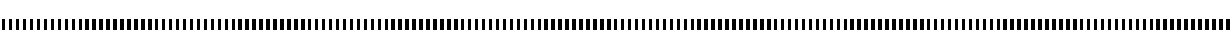

ARIMA Journal, vol. 17 (2014), pp. 93-117. 


\section{INTRODUCTION}

We call elastic waves, waves which propagate within elastic media. They can pass through a medium without modifying it durably. There are two main classes of elastic waves: body waves which travel through the volume of a material, and surface waves which travel along its surface. Concerning body waves, we can differentiate pressure waves ( $\mathrm{P}$-waves) which deform the medium in the same direction as their propagation direction, and shear waves ( $\mathrm{S}$ - waves) which deform the medium in the orthogonal direction to their propagation direction. In an anisotropic medium, we have two types of shear waves: $\mathrm{SH}$-waves with a horizontal polarisation, and the SV-waves with a vertical polarisation. Surface waves are the consequence of interactions of body waves with the free surface, and are constituted of a linear combinaison of these. We have two types of surface waves: Love waves which result from the combinaison of $\mathrm{P}$-waves with $\mathrm{SH}$-waves and Rayleigh waves which result of the combination of $\mathrm{P}$-waves with $\mathrm{SV}$-waves. Body waves are known to be non-dispersive, while surface waves are dispersive.

For the study of the propagation of waves in elastic media, it is more and more necessary to use numerical simulation, as the existence of analytical solutions is only valid in few cases like homogeneous domains with simple geometries. The modelling of this phenomenon is given by the wave equations of elastodynamic. Various numerical methods have been developped to solve such problems. Among them, we can mention the finite difference method [18][12], the classical finite element method [11][13], the spectral and pseudospectral methods [10], and the finite volume method [3].

We choose to use a high-order discontinuous Galerkin method (DG) applied to triangular meshes. The DG method was initially introduced by Reed and Hill for the solution of the neutron transport equation [15]. Neglected during many years, it is now very popular to solve hyperbolic problems. An advantage of this method is the fact that, due to the linearity of an equation, the solution can be computed element by element when the elements are suitably ordered, according to the characteristic direction. The discontinuity of the method provides a natural upwind. It had been used to solve nonlinear hyperbolic systems and convection-dominated problems, and it is now applied to various problems in applied mathematics [4]. It has shown its ability to capture strong discontinuties and shocks for solid and fluid mechanic problems [4], and it is now also used for the solution of different wave propagation problems, coupled with various time integration schemes. Inspite of its success in many domains of application, this method has been rarely applied to seismic wave propagation problems. Käser et al. ([9] and many references therein) proposed a DG finite element scheme based on upwind fluxes and the ADER approach in order to solve the elastodynamic system with the same high accuracy in space and time. Antonietti et al [2] compared the Mortar spectral element method and the DG spectral element method, both on non conforming rectangular meshes, with a second order leapfrog scheme for the time integration. They found that those two methods have a good accuracy while used for the simulation of the elastodynamic equations. Agut et al [1] developped a new high order method based on the "Modified Equation" technique in time, coupled with a DG method in space for the discretisation of the additional biharmonic operator, for the solution of the acoustic wave equations. Their results show that the computational cost of their scheme is the same as the one of the leapfrog scheme. In general, the advantages of DG methods are: 
- the ability to achieve a high order of accuracy by simply increasing the interpolation order of the basis functions locally in the elements,

- the fact that for an explicit scheme we do not have to invert a global linear system,

- the wide range of choice offered for the time integration scheme,

- the ability to have complex geometries and the possibility to use complex 3D meshes (including non conforming, mixed and locally refined meshes),

- the possibility to have different local interpolation orders within the elements [6],

- their capability of capturing discontinuities,

- the fact that they remain highly parallelizable.

Of course this has a cost in terms of computational time and memory usage, especially if we do not take care of the used time scheme. The DG methods developped in our team differ from the one used by Kaser and al by the use of centered flux within neigbouring elements and the choice of leapfrog schemes for the time integration. So we have a centered scheme in space and in time. The reason for this choice is that centered schemes are known to be non diffusive. This quality is important for the accurate approximation of a wave after a long time of propagation as it is the case for seismic simulations over the entired earth, and also for the conservation of the total elastodynamic energy. The preliminary results obtained with a leapfrog scheme of order 2 coupled with different interpolation orders of the discontinuous Galerkin scheme varying from one to four confirm all the benefits expected for this approach [5].

In this paper, we present the extension of the leapfrog time scheme from 2 up to an arbitrary even order for the elastodynamic equations, in order to have an arbitrary high order scheme both in space and time, and the results and performances of those schemes on academic and more realistic simulations. So the paper is structured as follows: In section 2, we introduce the governing equations in the velocity-stress formulation, with the boundary conditions used on the physical limits of the simulation domains in order to reproduce the real behaviour of the waves during their propagation. In section 3 , we present the spatial discretisation with the discontinuous Galerkin finite element method. In section 4 we present the connection of the DG scheme with the leapfrog scheme for the time integration. We present a description of the generation of leapfrog schemes of arbitrary high even order. In section 5, we show the application of the scheme on a two dimensional "eigenmode" homogeneous academic exemple, coupled with a numerical stability analysis, a numerical convergence analysis and a performance analysis on triangular regular and irregular meshes. And finally, section 6 is dedicated to the study of the two dimensional Garvin problem.

\section{EQUATIONS}

In a linear, isotropic and infinite medium, the P-SV wave propagation is modelled by the elastodynamic equation, which can be written in velocity-stress formulation [18], with the velocity and the stress as unknowns,

$$
\left\{\begin{aligned}
\rho \partial_{t} \vec{v} & =\operatorname{div}(\underline{\sigma}), \\
\partial_{t} \underline{\sigma} & =\lambda \operatorname{div}(\vec{v}) \underline{I}+\mu\left(\nabla \vec{v}+(\nabla \vec{v})^{T}\right),
\end{aligned}\right.
$$


where $\vec{v}$ is the velocity vector, $\sigma$ the stress tensor, $\rho$ the density of the medium, $I$ is the identity matrix and $\lambda$ and $\mu$ the Lamé coefficients describing the rheology of the medium, related to the P- and S-wave velocities by $V_{P}=\sqrt{(\lambda+2 \mu) / \rho}$ and $V_{S}=\sqrt{\mu / \rho}$.

System (1) is closed by adding physical boundary conditions at the free surface of the medium : $\underline{\sigma} \vec{n}=\overrightarrow{0}$, where $\vec{n}$ is the unity vector normal to the free surface. External forces are neglected.

Since the stress tensor is symmetrical, the unknown vector $\vec{W}$ may be written $\vec{W}=\left(v_{x}, v_{y}, \sigma_{x x}, \sigma_{y y}, \sigma_{x y}\right)^{t}$ and (1) expressed in matrix form by

$$
\partial_{t} \vec{W}-\sum_{m \in\{1,2\}} \underline{A}_{m}(\rho, \lambda, \mu) \partial x_{m} \vec{W}=0 .
$$

\section{SPATIAL DISCRETIZATION}

\subsection{Weak form of the problem}

As the velocity field $\vec{v}$ and the stress field $\vec{\sigma}$ are discontinuous, the weak form of the problem is written element by element. So let us note $\Omega_{i}$ an elementary $\mathbb{R}^{2}$ domain in which $\vec{W}$ ( $\vec{v}$ and $\vec{\sigma}$ ) is continuous everywhere, unless on the boundary, and let us note $L^{2}\left(\Omega_{i}\right)$ the Hilbert space of functions for which the squarre can be integrated on $\Omega_{i}$ : $L^{2}\left(\Omega_{i}\right)=\left\{v_{i} \in \mathbb{R}^{2}, \int_{\Omega_{i}} v_{i}^{2} d \Omega<\infty\right\}$, and $H^{1}\left(\Omega_{i}\right)$ the Sobolev space of $L^{2}\left(\Omega_{i}\right)$, defined by $H^{1}\left(\Omega_{i}\right)=\left\{v_{i} \in L^{2}\left(\Omega_{i}\right) \mid \nabla v_{i} \in\left(L^{2}\left(\Omega_{i}\right)\right)^{2}\right\}$.

The problem (2) can be written in the following form:

Search for $\tilde{\mathrm{W}}_{\mathrm{i}} \in\left(L^{2}\left(\Omega_{i}\right)\right)^{5}$, such that, $\forall \vec{\phi}_{k} \in\left(H^{1}\left(\Omega_{i}\right)\right)^{5}$ a test function, we have

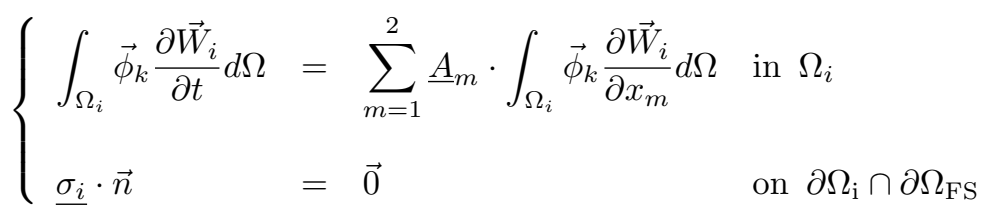

Now, we can use the Green theorem on the first equation of the system. We then obtain the weak form of the problem which is

Find $\tilde{\mathrm{W}}_{\mathrm{i}} \in\left(L^{2}\left(\Omega_{i}\right)\right)^{5}$, such that, $\forall \vec{\phi}_{k} \in\left(H^{1}\left(\Omega_{i}\right)\right)^{5}$, we have

$$
\int_{\Omega_{i}} \vec{\phi}_{k} \frac{\partial \vec{W}_{i}}{\partial t} d \Omega=\sum_{m=1}^{2} \underline{A}_{m} \cdot\left[-\int_{\Omega_{i}} \vec{W}_{i} \frac{\partial \vec{\phi}_{k}}{\partial x_{m}} d \Omega+\int_{\partial \Omega_{i}} \vec{\phi}_{k} \cdot\left(n_{m} \vec{W}_{i}\right) d \Gamma\right]
$$

where $\vec{n}_{i}$ is the outgoing unity vector normal to $\partial \Omega_{i}$.

We note that the consequence of the free surface condition is the vanishing of one part of the surface integration terms on the free surface of the domain. As $\underline{\sigma} \vec{n}=\overrightarrow{0}$ on $\partial \Omega_{F S}$. 


\subsection{Discretization using simplectic finite elements and a Lagrange polynomial basis}

For the discretization of the computational domain, we choose simplectic conforming finite elements (triangles in 2D). The interpolation functions used for the approximation of the field $W_{h}$ on every element are the Lagrange polynomials. The dimension of the Lagrange polynomial basis depends on the order of the interpolation chosen and on the space dimension. We define the set of Lagrange polynomials of degree $n$ on each element $\Omega_{i}$ by $P^{n}$. The basis functions are $\phi_{i}$ and are defined by $\phi_{i}\left(X_{j}\right)=\delta_{i j}$. The number $n_{k}$ of basis functions is $n_{k}=\frac{(n+1) \cdots(n+d)}{d}$, where $n$ is the order of the interpolation functions and $d$ the space dimension.

We then have the following approximations:

$$
\begin{aligned}
\Omega_{h} & =\bigcup \Omega_{i}, \\
\vec{W}_{h} & =\sum_{\Omega_{i} \in \Omega_{h}} \vec{W}_{i}, \\
\vec{W}_{i} & =\sum_{j=1}^{n_{k}} \vec{W}_{i j}, \\
\vec{W}_{i j} & =W_{i j} \vec{\phi}_{i j}, \\
\vec{\phi}_{i} & =\sum_{k=1}^{n_{k}} \alpha_{k} \vec{\phi}_{i k},
\end{aligned}
$$

where $n_{k}$ is the dimension of the Lagrange polynomial basis, and $\vec{\phi}_{i k}$ the vectors of that basis.

\subsection{Discrete weak form of the problem}

Let $\Omega_{h}$ be a conforming finite element triangulation of $\Omega$ and suppose that we have $\Omega_{h}=\bigcup_{i=1}^{N} \Omega_{i}$.

We define the following spaces:

$$
\mathcal{W}_{h}=\left\{\vec{W}_{h}=\sum_{\Omega_{i} \in \Omega_{h}} \vec{W}_{i} \mid \vec{W}_{i} \in\left(L^{2}\left(\Omega_{i}\right)\right)^{5}\right\}, \mathcal{V}_{h}=\left\{\vec{\phi}_{h}=\sum_{j=1}^{n k} \vec{\phi}_{i j} \mid \vec{\phi}_{i j} \in H^{1}\left(\Omega_{i}\right)\right\}
$$

The integrals of the weak form of the problem (4) can then be written in the following discrete form:

Find $\tilde{W}_{\mathrm{h}} \in \mathcal{W}_{\mathrm{h}}$ such that, $\forall \vec{\phi}_{h} \in \mathcal{V}_{h}$, we have

$$
\begin{aligned}
\sum_{j=1}^{n_{k}} \sum_{k=1}^{n_{k}} \frac{\partial \vec{W}_{i j}}{\partial t} \int_{\Omega_{i}} \vec{\phi}_{i k} \vec{\phi}_{i j} d \Omega= & \sum_{j=1}^{n_{k}} \sum_{k=1}^{n_{k}} \sum_{m=1}^{2} \underline{A}_{m} \cdot\left[-\vec{W}_{i j} \int_{\Omega_{i}} \vec{\phi}_{i j} \frac{\partial \vec{\phi}_{i k}}{\partial x_{m}} d \Omega\right. \\
& \left.+\int_{\partial \Omega_{i}}\left(\vec{\phi}_{i k} \cdot \vec{\phi}_{i j}\right)\left(n_{i_{m}} \vec{W}_{\left.i j\right|_{\partial \Omega_{i}}}\right) d \Gamma\right]
\end{aligned}
$$


The term $\vec{W}_{\left.i j\right|_{\partial \Omega_{i}}}$ has two different expressions, depending on whether $\partial \Omega_{i}$ is part of the boundary of the computational domain or not. For the term $\vec{W}_{\left.i j\right|_{\partial \Omega_{i} \cap \partial \Omega_{k}}}$ we use the following centrered approximation:

$$
\vec{W}_{i j \mid \partial \Omega_{i} \cap \partial \Omega_{k}}=\frac{\vec{W}_{i j}+\vec{W}_{k j}}{2}
$$

On the boundary of the computational domain we have the following approximation:

$$
\vec{W}_{\left.i\right|_{\partial \Omega_{i} \cap \partial \Omega_{h}}}=\vec{W}_{i}
$$

\subsection{Expression of the terms of the discrete form}

The first integral of the discrete form is the time integral. It can be express, using a scalar $B$ representing the integral of the scalar product of the basis functions, in the following form:

$$
\frac{\partial \vec{W}_{i j}}{\partial t} \int_{\Omega_{i}} \vec{\phi}_{i k} \cdot \vec{\phi}_{i j} d \Omega=B \frac{\partial \vec{W}_{i j}}{\partial t}
$$

Following the same approach, we can express the spatial integrals in the elements or in the intersection of two neighbouring elements as:

$$
\begin{aligned}
& \underline{A}_{m} \vec{W}_{i j} \int_{\Omega_{i}} \vec{\phi}_{i j} \frac{\partial \vec{\phi}_{i k}}{\partial x_{m}} d \Omega=G_{i_{\{j, k\}} m} \underline{A}_{m} \vec{W}_{i j}, \\
& \int_{\partial \Omega_{i} \cap \partial \Omega_{l}} \vec{\phi}_{i k}\left(\vec{W}_{i j \mid \partial \Omega_{i l}} n_{m}\right) d \Gamma= \int_{\partial \Omega_{i} \cap \partial \Omega_{l}} \vec{\phi}_{i k} \frac{1}{2}\left(\vec{W}_{i j} \cdot \vec{\phi}_{i j}+\vec{W}_{l j} \cdot \vec{\phi}_{l j}\right) n_{m} d \Gamma \\
&= \frac{1}{2}\left(\vec{W}_{i j} \int_{\partial \Omega_{i} \cap \partial \Omega_{l}} n_{m} \vec{\phi}_{i k} \cdot \vec{\phi}_{i j} d \Gamma\right. \\
&\left.+\vec{W}_{l j} \int_{\partial \Omega_{i} \cap \partial \Omega_{l}} n_{m} \vec{\phi}_{i k} \cdot \vec{\phi}_{l j} d \Gamma\right)
\end{aligned}
$$

\section{TIME DISCRETIZATION}

For the time discretization, we use leapfrog schemes, that are centered schemes, therefore not diffusive, and that are adapted to the mixed structure of the equations. Also, when combined with the flux, the resulting time domain scheme is non-dissipative [5]

We propose a leapfrog scheme derivation methodology that may give the temporal approximation to every even order desired for the elastodynamic problem, as Jeffrey $\mathrm{L}$. Young [19] and Spachmann et al [17] did for the Maxwell's equations.

The idea is to use the Taylor limited development, to estimate a centered approximation of the arbitrary even high order temporal derivative of the velocity field and of the stress field respectively.

For a scalar differentiable function $\mathrm{f}$, the Taylor development at time $n+1$ is given by the following centered expression: 


$$
\begin{aligned}
f^{n+1}(x)= & f^{n-1}(x)+2 \Delta t \frac{\partial f^{n}(x)}{\partial t}+2 \frac{\Delta t^{3}}{3 !} \frac{\partial^{3}\left(f^{n}(x)\right)}{\partial t^{3}}+\cdots \\
& +2 \frac{\Delta t^{2 N-1}}{(2 N-1) !} \frac{\partial^{2 N-1} f^{n}(x)}{\partial t^{2 N-1}}+O\left(\Delta t^{2 N+1}\right)
\end{aligned}
$$

So, for such a scheme and for a time step of $\Delta t$, we have to approximate the function every two time steps. The leapfrog approach applied to problem (1) simply consists in the solution of one of the two equations to odd time steps, and the other to even time steps. Another way to obtain the leapfrog scheme which is popular, is to divide the time step by 2 , and to use $\Delta t^{\prime}=\frac{\Delta t}{2}$ instead of $\Delta t$. We then evaluate the first equation of the elastodynamic system at time steps $n \Delta t+1 / 2$, and the second equation of the system at time steps $n \Delta t$. In our case, we chose this second approach. Finally, we have to evaluate the successive derivatives of the function up to the desired order.

\subsection{Construction of an arbitrary high order leapfrog scheme for the continuous elastodynamic problem}

Let us apply the previous discretization to the elastodynamic problem (1). We decide to divide the normal time step by two and to calculate the velocity field at time step $n+1 / 2$, and the stress field at time step $n$. Finally, the scheme obtained has the following form:

$$
\left\{\begin{aligned}
\vec{v}^{n+1 / 2}= & \vec{v}^{n-1 / 2}+\Delta t \frac{\partial \vec{v}^{n}}{\partial t}+\frac{\Delta t^{3}}{24} \frac{\partial^{3} \vec{v}^{n}}{\partial t^{3}}+\cdots \\
& +\frac{\Delta t^{2 N+1}}{2^{2 N}(2 N+1) !} \frac{\partial^{2 N+1} \vec{v}^{n}}{\partial t^{2 N+1}}+O\left(\Delta t^{2 N+3}\right) \\
\vec{\sigma}^{n+1}= & \vec{\sigma}^{n}+\Delta t \frac{\partial \vec{\sigma}^{n+1 / 2}}{\partial t}+\frac{\Delta t^{3}}{24} \frac{\partial^{3} \vec{\sigma}^{n+1 / 2}}{\partial t^{3}}+\cdots \\
& +\frac{\Delta t^{2 N+1}}{2^{2 N}(2 N+1) !} \frac{\partial^{2 N+1} \vec{\sigma}^{n+1} 2^{2 N}}{\partial t^{2 N+1}}+O\left(\Delta t^{2 N+3}\right)
\end{aligned}\right.
$$

We then have to approximate the successive derivatives of the velocity field and of the stress field up to the desired order, using the mixed structure of equation (1) which can be written in the following form:

$$
\left\{\begin{aligned}
\frac{\partial \vec{v}^{n+1 / 2}}{\partial t} & =f\left(\vec{\sigma}^{n}\right) \\
\frac{\partial \vec{\sigma}^{n+1}}{\partial t} & =g\left(\vec{v}^{n+1 / 2}\right)
\end{aligned}\right.
$$

- Construction of the second order leapfrog scheme

We have to evaluate $\frac{\partial \vec{v}^{n}}{\partial t}$ and $\frac{\partial \vec{\sigma}^{n+\frac{1}{2}}}{\partial t}$.

For that purpose, from equations (8), we obtain:

$$
\left\{\begin{aligned}
\vec{T}_{1} & =\Delta \operatorname{tf}\left(\vec{\sigma}^{n}\right) \\
\vec{T}_{1}^{\prime} & =\Delta \operatorname{tg}\left(\vec{v}^{n+1 / 2}\right) \\
\vec{v}^{n+1 / 2} & =\vec{v}^{n-1 / 2}+\vec{T}_{1} \\
\vec{\sigma}^{n+1} & =\vec{\sigma}^{n}+\vec{T}_{1}^{\prime}
\end{aligned}\right.
$$


As this time discretization scheme is only second order accurate, the global accuracy of the scheme can be penalized when higher-degree polynomials $(m>2)$ are used for spatial approximation [5]. Then, we propose a higher-order leapfrog scheme following the method proposed for the Maxwell's equations by Young [19] or Spachmann et al. [17] and applied to a DG method by Fahs [6].

- Construction of the fourth order leapfrog scheme

We have to evaluate $\frac{\partial^{3} \vec{v}^{n}}{\partial t^{3}}$ and $\frac{\partial^{3} \vec{\sigma}^{n+1 / 2}}{\partial t^{3}}$.

For that purpose, we use equation (7) and equation (8). We then obtain:

$$
\left\{\begin{aligned}
\vec{T}_{1} & =\Delta t f\left(\vec{\sigma}^{n}\right) \\
\vec{T}_{2} & =\Delta t g\left(\vec{T}_{1}\right) \\
\vec{T}_{3} & =\Delta t f\left(\vec{T}_{2}\right) \\
\vec{v}^{n+1 / 2} & =\vec{v}^{n-1 / 2}+\vec{T}_{1}+\frac{\vec{T}_{3}}{24} \\
\vec{T}_{1}^{\prime} & =\Delta t g\left(\vec{v}^{n+1 / 2}\right) \\
\vec{T}_{2}^{\prime} & =\frac{\Delta t}{\rho} f\left(\vec{T}_{1}^{\prime}\right) \\
\vec{T}_{3}^{\prime} & =\Delta t g\left(\vec{T}_{2}^{\prime}\right) \\
\vec{\sigma}^{n+1} & =\vec{\sigma}^{n}+\vec{T}_{1}^{\prime}+\frac{\vec{T}_{3}^{\prime}}{24}
\end{aligned}\right.
$$

- Construction of the arbitrary high even order leapfrog scheme

We generalize the technique shown in the previous paragraph, and we obtain, for an arbitrary even order $2 \mathrm{~N}$,

$$
\left\{\begin{aligned}
\vec{T}_{0} & =\vec{\sigma}^{n} & & \\
\vec{T}_{2 k+1} & =\Delta t f\left(\vec{T}_{2 k}\right) & & \forall k \geq 0 \\
\vec{T}_{2 k} & =\Delta t g\left(\vec{T}_{2 k-1}\right) & & \forall k \geq 1 \\
\vec{v}^{n+1 / 2} & =\vec{v}^{n-1 / 2}+\sum_{k=1}^{N} \frac{\vec{T}_{2 k-1}}{(2 k-1) ! 2^{2 k-2}} & & \text { at order } 2 \mathrm{~N} \\
\vec{T}_{0}^{\prime} & =\vec{v}^{n+1 / 2} & & \\
\vec{T}_{2 k+1}^{\prime} & =\Delta t g\left(\vec{T}_{2 k}^{\prime}\right) & & \forall k \geq 0 \\
\vec{T}_{2 k}^{\prime} & =\Delta t f\left(\vec{T}_{2 k-1}^{\prime}\right) & & \\
\vec{\sigma}^{n+1} & =\vec{\sigma}^{n}+\sum_{k=1}^{N} \frac{\vec{T}_{2 k-1}^{\prime}}{(2 k-1) ! 2^{2 k-2}} & & \text { at order } 2 \mathrm{~N}
\end{aligned}\right.
$$

\subsection{Application of the arbitrary high order leapfrog scheme on the discontinuous Galerkin spatial discretization for the elastodynamic equations}

The consequence of the use of the leapfrog scheme is that we have to split the vector $W$ of unknowns and then solve the two equations at different times: the velocity unknowns are evaluated at time $n+1 / 2$ and the stress unknowns are evaluated at time $n$. So the spatial discretization of the equations, coupled with the time scheme has the following form for the velocity equation: 


$$
\begin{aligned}
\int_{\partial \Omega_{i}} \phi_{i j} \frac{\vec{v}^{n+\frac{1}{2}}-\vec{v}^{n-\frac{1}{2}}}{\Delta t} d \Omega= & \sum_{l=1}^{N / 2} \frac{\Delta t^{2 l-2}}{2^{2 l-2}(2 l-1) !} \int_{\partial \Omega_{i}} \phi_{i j} \frac{\partial^{2 l-1} \vec{v}^{n}}{\partial t^{2 l-1}} d \Omega \\
= & \int_{\partial \Omega_{i}} \phi_{i j} f\left(\vec{\sigma}^{n}\right) d \Omega+\cdots \\
& +\frac{\Delta t^{2 l-2}}{2^{2 l-2}(2 l-1) !} \int_{\partial \Omega_{i}} \phi_{i j} f\left(\frac{\partial^{2 l-2} \vec{\sigma}^{n}}{\partial t^{2 l-2}}\right) d \Omega
\end{aligned}
$$

For the stress equation, we have the following form:

$$
\begin{aligned}
\int_{\partial \Omega_{i}} \phi_{i j} \frac{\vec{\sigma}^{n+1}-\vec{\sigma}^{n}}{\Delta t} d \Omega= & \sum_{l=1}^{N / 2} \frac{\Delta t^{2 l-2}}{2^{2 l-2}(2 l-1) !} \int_{\partial \Omega_{i}} \phi_{i j} \frac{\partial^{2 l-1} \vec{\sigma}^{n+1 / 2}}{\partial t^{2 l-1}} d \Omega \\
= & \int_{\partial \Omega_{i}} \phi_{i j} g\left(\vec{v}^{n+1 / 2}\right) d \Omega+\cdots \\
& +\frac{\Delta t^{2 l-2}}{2^{2 l-2}(2 l-1) !} \int_{\partial \Omega_{i}} \phi_{i j} g\left(\frac{\partial^{2 l-2} \vec{v}^{n+1 / 2}}{\partial t^{2 l-2}}\right) d \Omega
\end{aligned}
$$

The weak form of those equations are then written using the Green theorem.

In practice, for a given approximation in space, the fourth-order leapfrog scheme needs three times the number of arithmetic operations (to calculate the fluxes $F$ and $G$ ) than the classical leapfrog scheme and twice as much memory storage since additional arrays have to be defined. And for the memory storage of the arbitrary high order scheme, we just have to forecast two additionnal arrays for $\vec{T}_{2 k}$ and $\vec{T}_{2 k+1}$ to the second-order scheme. So, the memory required for the leapforg scheme of order $N$ is the same as for the leapfrog scheme of order four.

\section{APPLICATION TO THE EIGENMODE HOMOGENEOUS PROBLEM}

A first application of our scheme has been carried out on the eigenmode homogeneous problem. In this exemple, the system is initialised with a sinusoidal wave on the unity square domain, with the free surface condition applied at all its boundary surface. We consider the $(1,1)$ mode whose exact solution is [8]

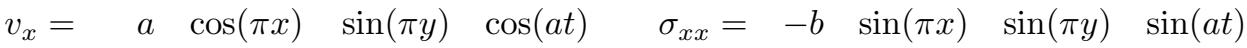

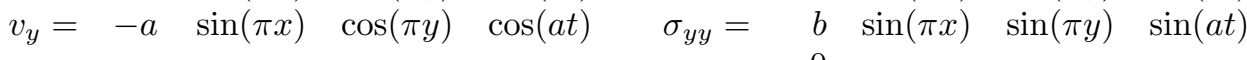

$$
\begin{aligned}
& \sigma_{x y}=0
\end{aligned}
$$

where $a=\sqrt{2} \pi V_{s}$ and $b=2 \pi \mu$.

The homogeneous material parameters are $\rho=1, \lambda=0.5$ and $\mu=0.25$, leading to the constant wave propagation velocities of $v_{p}=1$ and $v_{s}=0.5$, for $\mathrm{P}$-waves and $\mathrm{S}$-waves respectively. The initial condition is $v_{x}^{0}=\alpha \sin (\vec{k} \cdot \vec{x})+\beta \sin (\vec{k} \cdot \vec{x})$, with the wave number $\vec{k}=6 \pi(1,1)^{t}$. The initialisation of the leapfrog scheme is done from the 
exact solution (14) at $t=0$ for $v$ and $t=\frac{\Delta t}{2}$ for $\sigma, \Delta t$ being the time step of the scheme. The notation DGk-LFi $(\mathrm{k}=2, \ldots, 4$ and $\mathrm{i}=2$ or 4$)$ refers to a spatial discretization based on a polynomial basis of degree $k$ and a classical second-order leapfrog time scheme (LF2) or its extension (LF4).

\subsection{Numerical study of the stability}

In table (1) we present the evolution of the numerical CFL value of the program for the DG2 space interpolation as a function of the time scheme. These results show that the CFL value is multiplied by about 2.6 when we use the leapfrog scheme of order four instead of the leapfrog scheme of order two, both on regular and irregular meshes.

\begin{tabular}{|c|c|c|}
\hline Time scheme associated with the DG space discretisation & LF2 & LF4 \\
\hline CFL coefficient for the DG2 scheme on structured meshes & 0,2322 & 0,5928 \\
\hline CFL coefficient for the DG2 scheme on unstructured meshes & 0,2322 & 0,5928 \\
\hline CFL coefficient for the DG3 scheme on structured meshes & 0,1498 & 0,3821 \\
\hline CFL coefficient for the DG3 scheme on unstructured meshes & 0,1498 & 0,3821 \\
\hline CFL coefficient for the DG4 scheme on structured meshes & 0,0939 & 0,2644 \\
\hline CFL coefficient for the DG4 scheme on unstructured meshes & 0,0939 & 0,2644 \\
\hline
\end{tabular}

Table 1. Evolution of the CFL value for the DG space discretisations as a function of the order of the leapfrog time scheme

The figure 1 shows the evolution of the numerical CFL value of the program for a given time scheme, as a function of the DG space interpolation order. This figure shows that the evolution of the CFL value of the program is linear when we increase the polynomial interpolation order from two to three and from three to four.

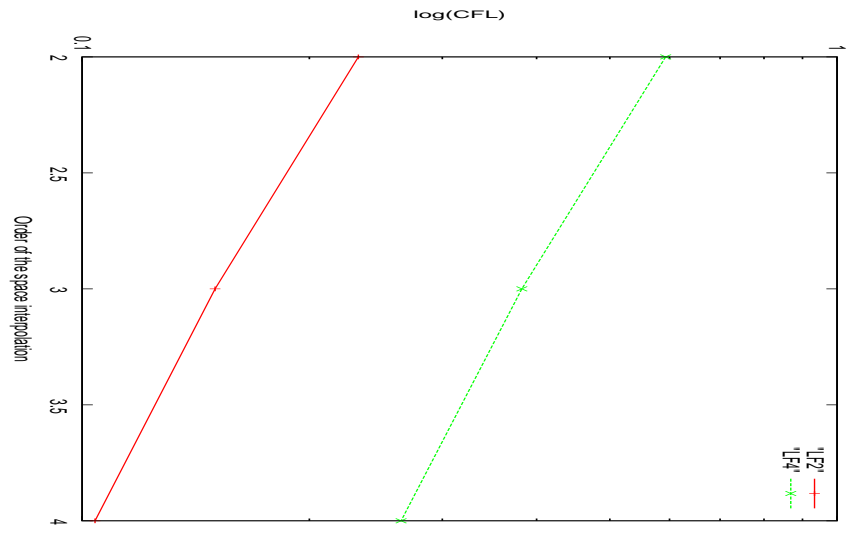

Figure 1. Evolution of the numerical CFL for a given leapfrog scheme 


\subsection{Numerical study of the convergence and CPU time analysis}

In this section, we numerically determine the convergence orders of the proposed DG-LF schemes without the source term. As test case, we solve the two dimensional homogeneous elastic wave equations on the unity square domain, with a sinusoidal periodic initial condition and four free surface boundaries (eigenmode problem), as presented in the previous section. The numerical experiments to determine the convergence orders of the schemes are performed on two sequences of triangular regular and irregular meshes. Each sequence consisting of four meshes with decreasing width. For the regular meshes, the refinement is controlled by multiplying by 2 the number of mesh elements in each direction. For the irregular meshes, we use the so-called red - refinement, where each triangle is refined subsequently by dividing it into four similar subtriangles. We characterize the refinement state of a mesh by the minimum edge of all the triangular elements.

\begin{tabular}{|c|c|c|}
\hline Scheme & Order of conv. for struct. meshes & Order of conv. for unstruct. meshes \\
\hline DG2/LF2 & 2.44 & 2.57 \\
\hline DG2/LF4 & 3.04 & 2.92 \\
\hline DG3/LF2 & 2.07 & 2.42 \\
\hline DG3/LF4 & 3.5 & 3.03 \\
\hline DG4/LF2 & 2.00 & 2.01 \\
\hline DG4/LF4 & 4.47 & 4.01 \\
\hline
\end{tabular}

Table 2. Value of the order of convergence for the different time/space schemes on structured meshes

In figure 2, we present the convergence curves of the DG2, DG3 and DG4 schemes, coupled with the leapfrog scheme of orders two and four (LF2 and LF4) for structured and unstructured meshes. The respective values of the orders of convergence are summarized in table 2. An analysis of the LF2 schemes shows that the order of convergence is about 2 for all of them. That shows that the theoretical order of convergence given by the DG space discretization is not reached. This affirmation is confirmed by the curves of figure 3 and figure 4 for structured and unstructured meshes, which show an increase of the order of convergence for those schemes while using the leapfrog scheme of order four. The leapfrog of order four scheme achieves a higher order of convergence than the leapfrog scheme of order two in terms of orders of convergence, level of the $L^{2}$ error, stability limits, and CPU time. Indeed, we can notice that the increase of the stability limits while using the LF4 scheme compensates the additional CPU time necessary to compute this scheme from the LF2 scheme. Therefore we observe a small decrease of the CPU time as shown in figure 5. An analysis of figure 6 shows that the gain obtained while increasing the time scheme is more important, as the level of the $L^{2}$ error is also more important for the 2nd-order leapfrog scheme. This implies that, to have a 2nd-order leapfrog scheme equivalent for a given 4th-order leapfrog scheme, we must proceed to an important coarsening of the mesh of the 4th-order leapfrog scheme. Figure 7 also shows that the 4th-order leapfrog scheme coupled with the DG3 interpolation in space is equivalent in terms of cpu time to the 2nd-order leapfrog scheme coupled with the DG2 interpolation in space, and so are the 4th-order leapfrog scheme coupled with the DG4 interpolation in space and the 2nd-order leapfrog scheme coupled with the DG3 interpolation in space. But in terms of level of the $L^{2}$ error, figure 8 shows that the 4th- 
order leapfrog schemes are better than their equivalent 2 nd-order leapfrog schemes. This remark suggests that we need less points per wavelength if we increase the order of the time scheme. Finally, we can state that the developped schemes achieve the expected convergence orders on both regular and irregular meshes.

\subsection{Numerical verification of the conservation of the energy}

The study of the conservation of the energy property is very important for every scheme used for the numerical simulation of the propagation of elastic waves in a medium. For that, we computed the $L^{2}$ error of the scheme on the eigenmode problem, during 100 periods ( 300 seconds). The goal was to see the levels and the slopes of the error curves. As we can see in Figures 9 and Figure 10, the level of the errors decreases when the order of the scheme increases in time for a given space interpolation, or when the order of the space scheme increases for a given leapfrog scheme. Thus, we can observe that, for the DG2 and the DG3 interpolation, coupled with the 4th-order leapfrog scheme, the slopes of the error curves are nearly zero. This means that the level of the initial error, which is already very small, is preserved along the whole simulation. For the DG4 interpolation coupled with the order 4 leapfrog scheme, we observe a slight slope for the error curves. This is certainly due to the fact that we need to increase the order of the time scheme. Also, we can conclude that the schemes are numerically nearly non dissipative. This result is in agreement with the theory (our schemes are centered).

\section{STUDY OF THE GARVIN PROBLEM}

After the eingenmode problem, we studied a problem with an outgoing wave boundary condition: the Garvin test. The Garvin problem [7] is a classical test which permits to verify the accuracy of a numerical simulation software of elastic wave propagation. This problem is a simulation in a homogeneous elastic half space, with an explosive source. There is an analytical solution to this problem [16]. If the source is inside the medium, only a direct $\mathrm{P}$-wave is created and propagates in the medium. At the free surface of the medium, there is a reflection $\mathrm{P}-$ wave and a conversion $\mathrm{P}-\mathrm{S}$ wave which are created. If the source is directly under the free surface, there is the creation and the propagation of a strong Rayleigh wave along the free surface. In this case the Rayleigh wave is non dispersive, as the medium is homogeneous and its surface plane.

For our simulations, we are in this second case. The medium is a rectangular domain of $400 \mathrm{~m}$ by $200 \mathrm{~m}$. The three surface sensors are located at the epicenter, and at $45 \mathrm{~m}$ and $95 \mathrm{~m}$ from the first sensor respectively, as we can see in Figure (11). The material parameters of the medium are $\rho=1 \mathrm{~kg} / \mathrm{m}^{3}$ for the density, $v_{p}=103,92 \mathrm{~m} . \mathrm{s}^{-1}$ and $v_{s}=60 \mathrm{~m} \cdot \mathrm{s}^{-1}$ for the velocities of $P$-waves and $S$-waves respectively. The explosive source, which is located at a $1 \mathrm{~m}$ depth, is a Ricker having $4 \mathrm{~Hz}$ for its central frequency and $12 \mathrm{~Hz}$ for its maximum frequency:

$$
s(t)=\left[-1+2 a(t-0,3)^{2}\right] \exp \left[-a(t-0,3)^{2}\right]
$$

The coefficient $a$ has been set to 159.42 , and the wavelength is approximately equal to $5 \mathrm{~m}$. This source term is introduced as a right hand side on $\sigma_{x x}$ and $\sigma_{y y}$. Initial conditions for the system are $\mathrm{V}=0$ and $\sigma=0$ and solutions are computed until $\mathrm{t}=2.5 \mathrm{~s}$. Our objective is 

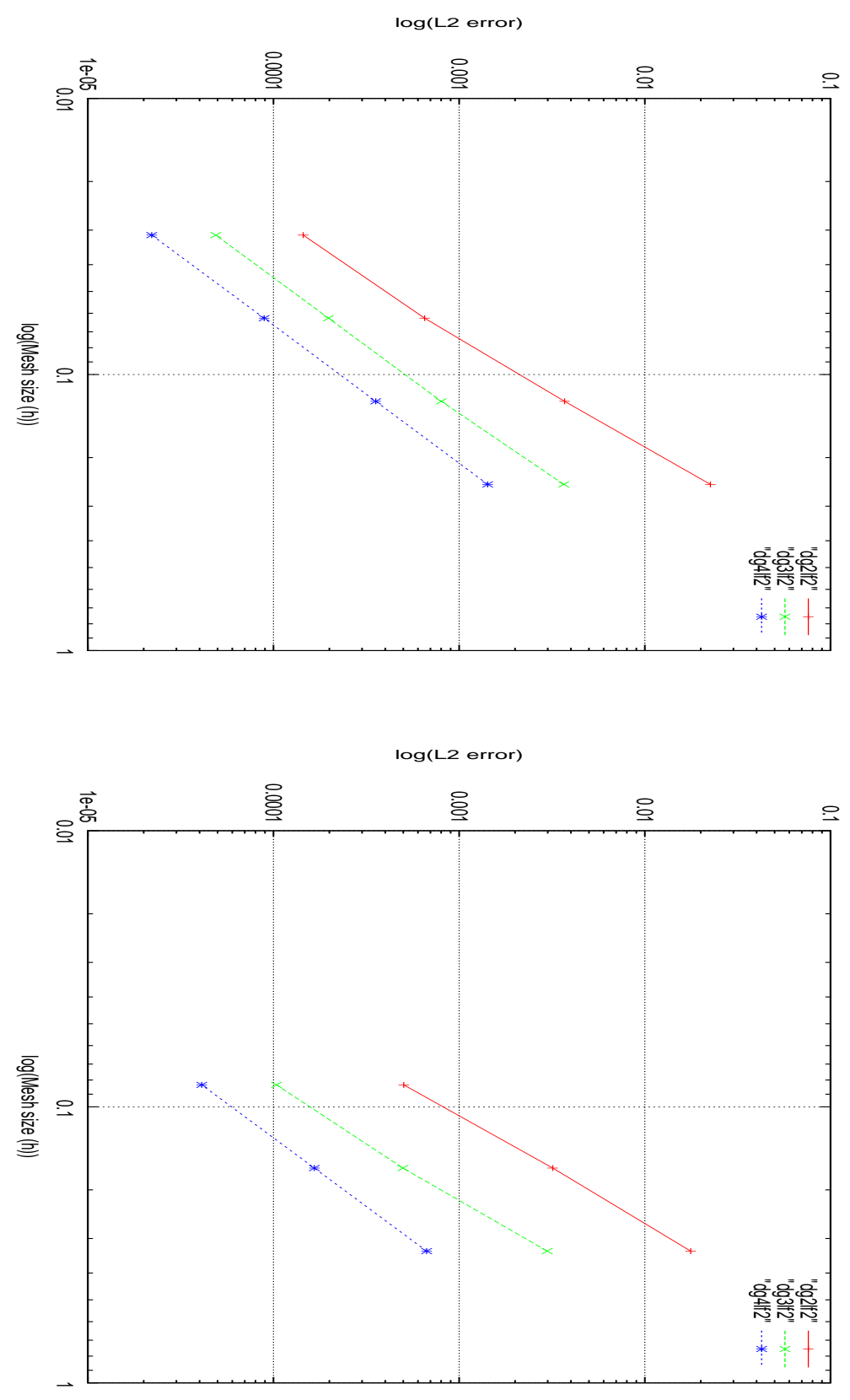

Figure 2. Convergence curves for LF2 leapfrog time schemes on structured (top) and unstructured meshes

to compare the results obtained with the DG2-LF2 and DG4-LF4 methods. The analytical solution is computed using a software written by Sanchez-Sesma and Perez-Rocha [16]. 
106 A R I M A - Volume $17-2014$
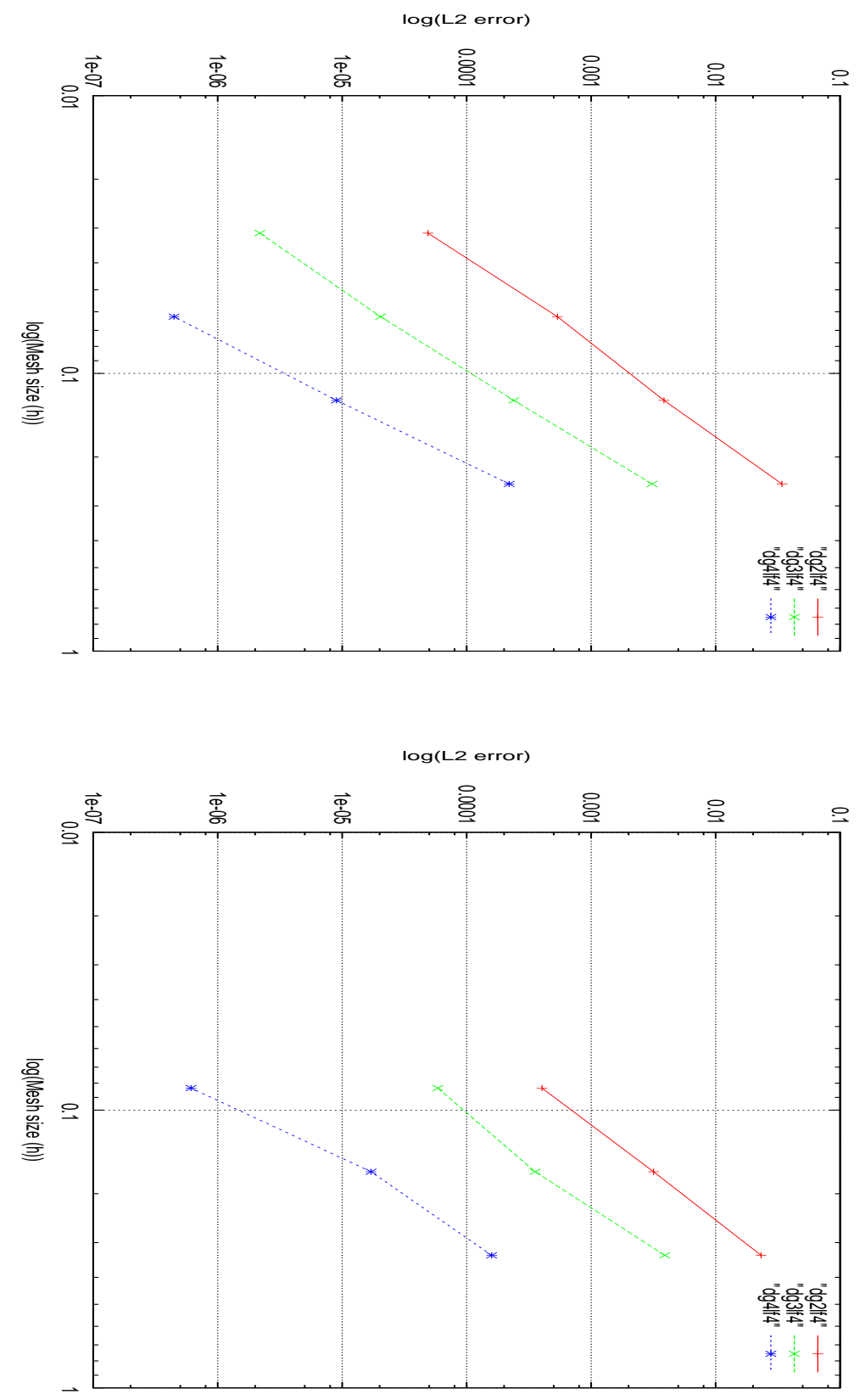

Figure 3. Convergence curves for LF4 leapfrog time schemes on structured (top) and unstructured meshes

To highlight the difference of both methods, we first use two uniform and very coarse meshes : M1 with $\mathrm{h}=5.0 \mathrm{~m}$ and containing 3600 triangles and $\mathrm{M} 2$ with $\mathrm{h}=2.5 \mathrm{~m}$ and 

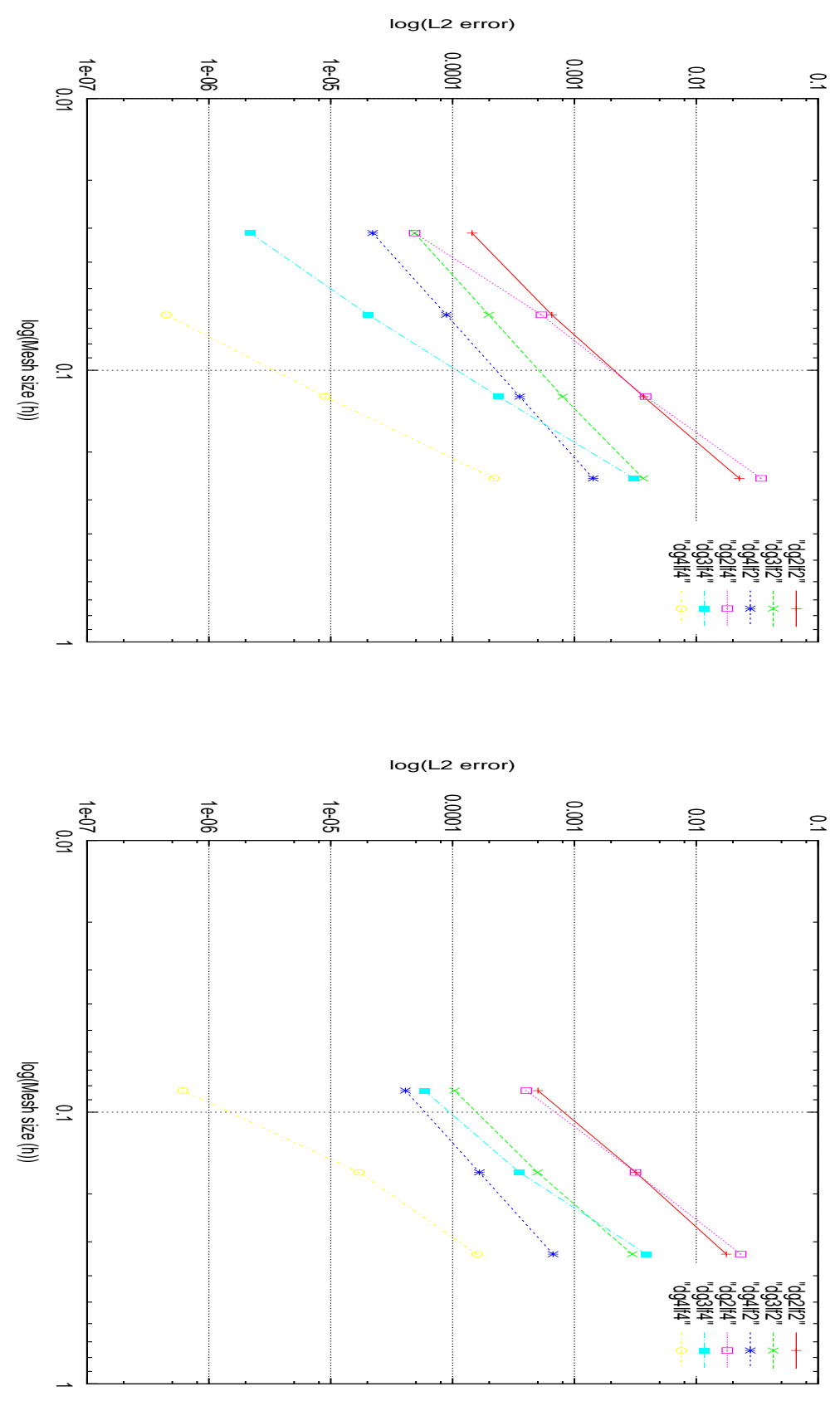

Figure 4. Convergence curves for the LF2 and the LF4 leapfrog time schemes on structured (top) and unstructured meshes 


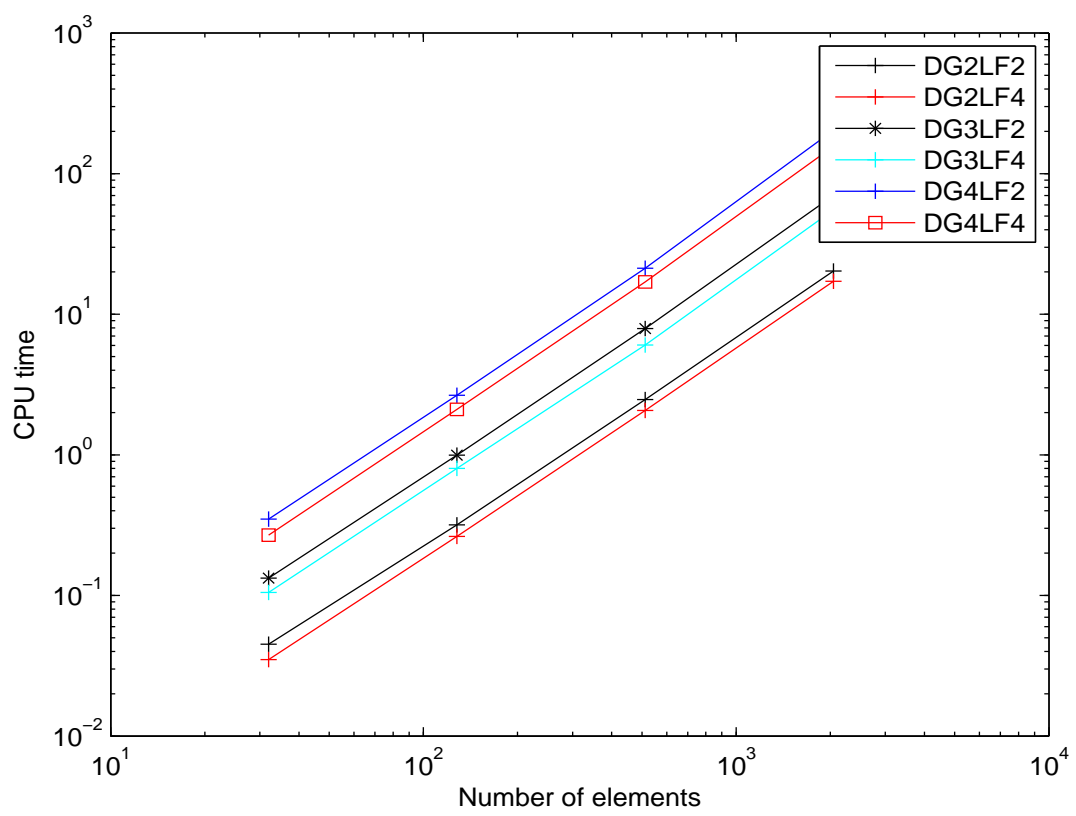

Figure 5. Evolution of the CPU time as a function of the number of elements for a given space/time scheme

14400 triangles. We define an error (in \%) between the solutions and the reference : for a given sensor and a component of the velocity, the error is the mean value of the relative errors calculated at the extreme of the profiles (between 3 and 6 values, as seen on Figures 12 to 14). We plot, in Figures 12, 13 and 14 the profiles of the velocity in the $\mathrm{y}$ direction $\left(v_{y}\right)$ as a function of time obtained at the three sensors and for the two coarse meshes M1 and M2 (first and second lines of all figures). Note that, for a better visibility, the solutions obtained at the three surface sensors have been plotted for different time windows. Observing the different figures, it is obvious that the DG4-LF4 method produces better solutions than the DG2-LF2 method. For the coarsest mesh M1, the profiles of the DG4-LF4 method contain all the characteristics of the reference solution. The results obtained with the mesh M2 are clearly closer to the reference solution but it still remains an important delay on the profile corresponding to the DG2-LF2 method at sensor $\mathrm{C} 1$. Finally, we introduce a finer mesh, M3 for which $\mathrm{h}=1.0 \mathrm{~m}$ and containing 90000 triangles. We plot, in Figures 12 to 14 (last line), the solutions obtained with the DG4-LF4 method. Except a slight delay of the solutions, especially at sensor C2, the results constitute a validation of our method. The values of the mean relative errors on $v_{y}$, obtained for the different schemes and meshes are given in Table 3. From the observations of the table, we notice that the errors on the amplitude of the solutions of the DG4-LF4 method are lower than those of the DG2-LF2 method, at all sensors and with the three meshes since the values of the mean error are divided by 2 . For the finest mesh $\mathrm{M} 3$, corresponding to a mesh spacing equal to $\Lambda / 5$, the error level is satisfactory. We also remark that the convergence towards the reference solution is slower than expected, 


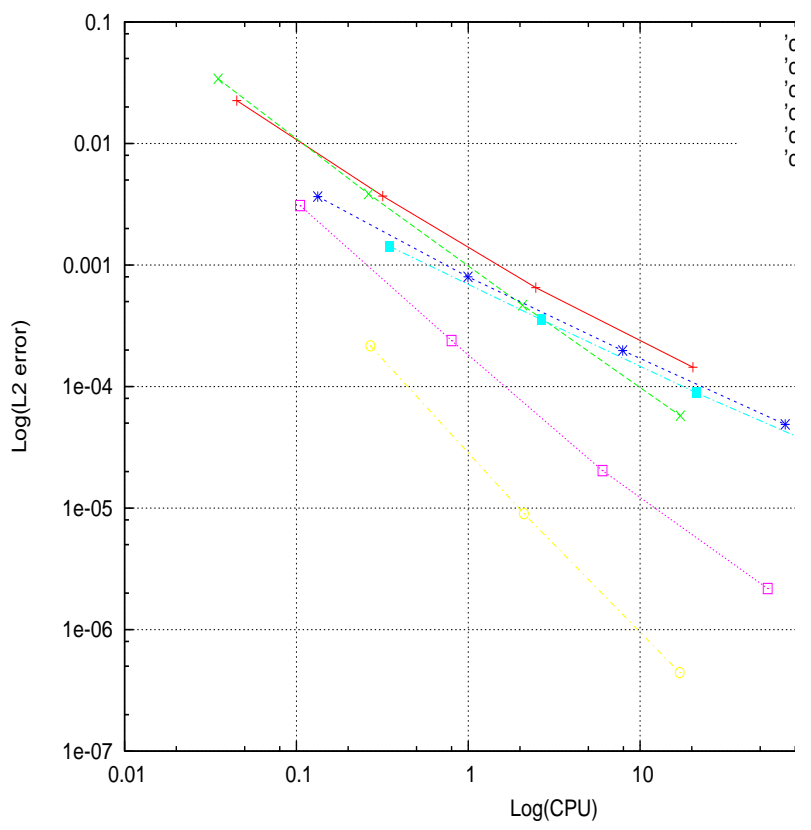

Figure 6. Evolution of the $L^{2}$ error in function of the CPU time

\begin{tabular}{|c|c|c|}
\hline & DG2-LF2 & DG4-LF4 \\
\hline C1-M1 & 10 & 47 \\
\hline C1-M2 & 60 & 10 \\
\hline C1-M3 & 10 & 5 \\
\hline \hline C2-M1 & 50 & 12 \\
\hline C2-M2 & 26 & 12 \\
\hline C2-M3 & 10 & 4 \\
\hline \hline C3-M1 & 63 & 24 \\
\hline C3-M2 & 33 & 12 \\
\hline C3-M3 & 10 & 6 \\
\hline
\end{tabular}

Table 3. Mean relative error (in \%) on vy at the three sensors 
110 A R I M A - Volume $17-2014$

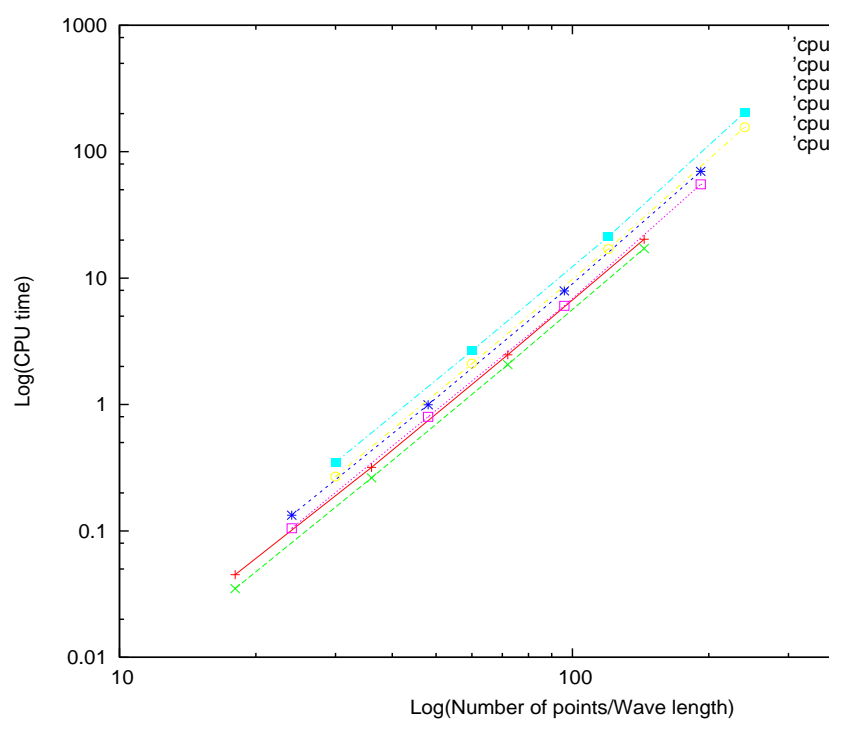

Figure 7. Evolution of the CPU time in function of the number of points/wave length for a given space/time scheme

especially when compared to the results of the eigenmode problem. It is probably due to the absorbing condition which is a basic upwind scheme and would gain to be improved.

\section{CONCLUSION}

We have presented the extention of our explicit leapfrog time scheme from a secondorder to an arbitrary high even order. This scheme seems to be stable under a CFL-like condition. Numerical results on two problems are presented to illustrate the ability of the scheme on a test case with free surface and outgoing wave boundary conditions. The results on the eigenmode exemple are given with a numerical stability analysis of the scheme and a numerical convergence study. These studies show the gains obtained by increasing the order of the time scheme in terms of increasing the CFL limit, decreasing of the $L^{2}$ error, decreasing of the CPU time.The results also show that the expected convergence rates are reached both on regular and irregular meshes, and that the scheme is robust and highly acurate, even on problems with free surfaces and outgoing waves boundary conditions. 


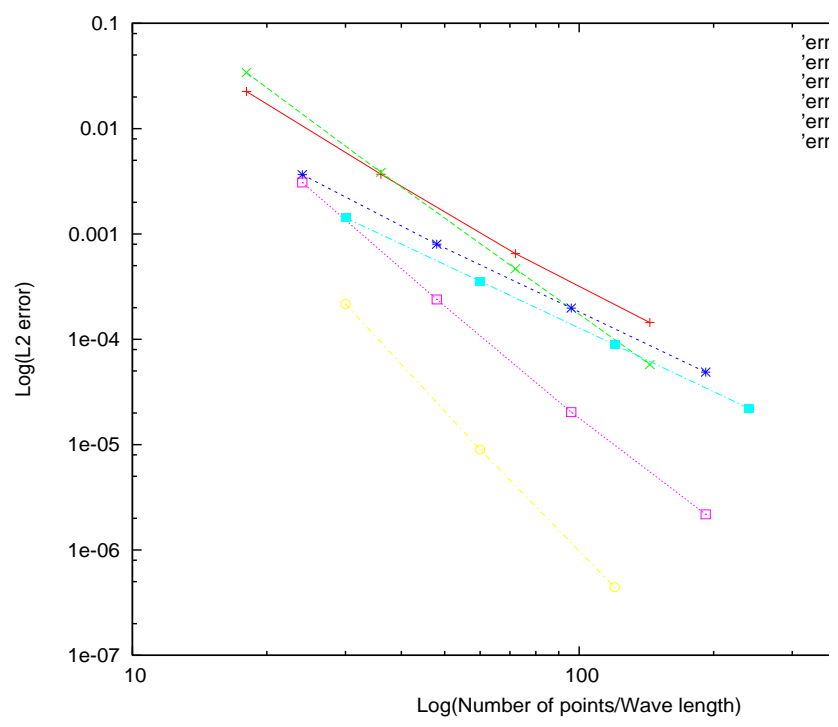

Figure 8. Evolution of the $L^{2}$ error as a function of the number of points/wave length for a given space/time scheme

\section{Acknowledgment}

The author thanks the AUF (Agence Universitaire de la Francophonie) for its financial support which permitted the realization of this work. He also thanks the NACHOS team of INRIA-Sophia Antipolis-Méditerranée in which this work had been performed and had been first published as an INRIA research report, expecially Nathalie GLINSKY, Stéphane LANTERI and Sarah DELCOURTE for their constant help and their precious advices.

\section{References}

[1] C. Agut, J. Diaz and Abdelaziz Ezziani, "High-Order Schemes Combining the Modified Equation Approach and Discontinuous Galerkin Approximations for the Wave Equation", Commun. Comput. Phys., Vol. 11, No. 2, pp. 691-708 February 2012. 
112 A R I M A - Volume $17-2014$
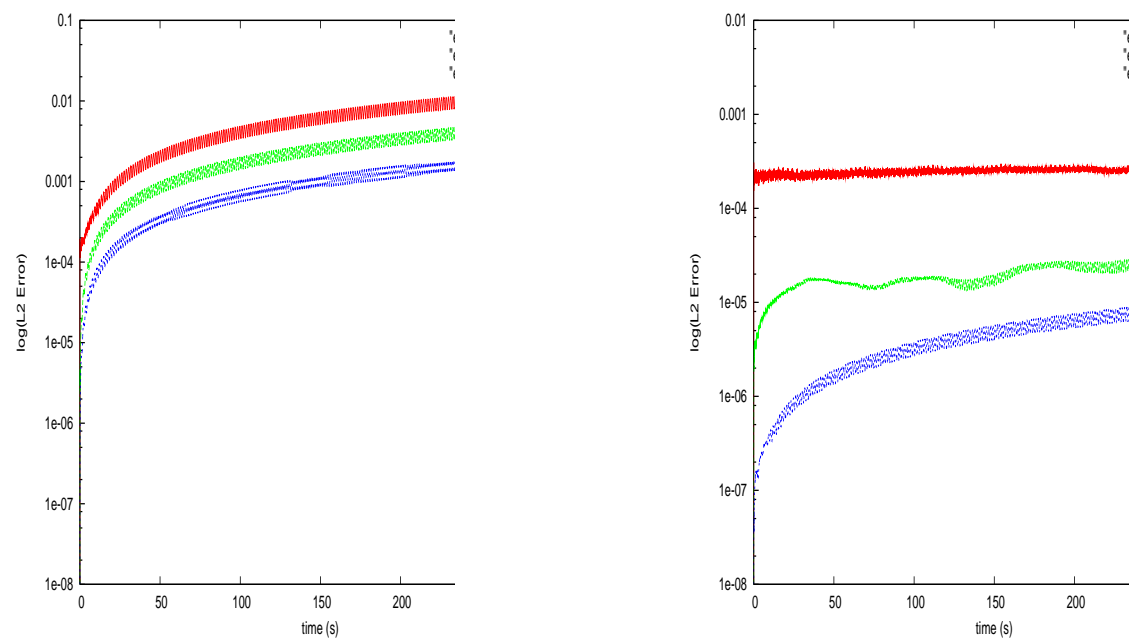

Figure 9. Evolution of the $L^{2}$ error of the solution on 100 periods for the LF2 scheme (left) and for the LF4 scheme (right)

[2] P.F. Antonietti, I. Mazzieri, A. Quarteroni, F. Rapetti, "Non-conforming high order approximations of the elastodynamics equation", Comput. Methods Appl. Mech. Engrg. 209212 (2012) 212-238, 2012.

[3] M. BenjemaA, N. Glinsky-Olivier, V. M. Cruz-Atienza, J. Virieux and S. PIPERNO, "Dynamic non-planar crack rupture by a finite volume method", Geophys. J. Int. 209-212 (2007) 212-238, vol. 171, 271-285, 2007.

[4] B. COCKBURN "Discontinuous Galerkin methods for convection-dominated problems. Highorder methods for computational physics", 69-224, Lect. Notes Comput. Sci. Eng., vol. 9, Springer, Berlin, 1999

[5] S. Delcourte, L. Fezoui and N. Glinsky-Olivier, "A high-order discontinuous Galerkin method for the seismic wave propagation", ESAIM : Proceedings, num. 27, 2009.

[6] H. FAHS, "Méthode de type Galerkin discontinu d'ordre élevé pour la résolution numérique des équations de Maxwell instationnaires sur des maillages simplexes non-conformes", Thèse de doctorat de l'Université de Nice-Sophia Antipolis, 2008.

[7] W. Garvin, "Exact transient solution of the buried line source problem", Proc. R. Soc. London ser. A, num. 234, 528-541, 1956

[8] N. Glinsky, S. Moto Mpong And S. Delcourte, “A High-order Discontinuous Galerkin Scheme for Elastic Wave Propagation”, Rapport de recherche INRIA, num. 7476, 2010.

[9] M. KÄSER, V. HERMAnN AND J. DE LA PUENTE, "Accuracy alalysis of the Discontinuous Galerkin method for seismic wave propagation”, Geophys. J. Int., vol. 173, num. 3, 2008. 


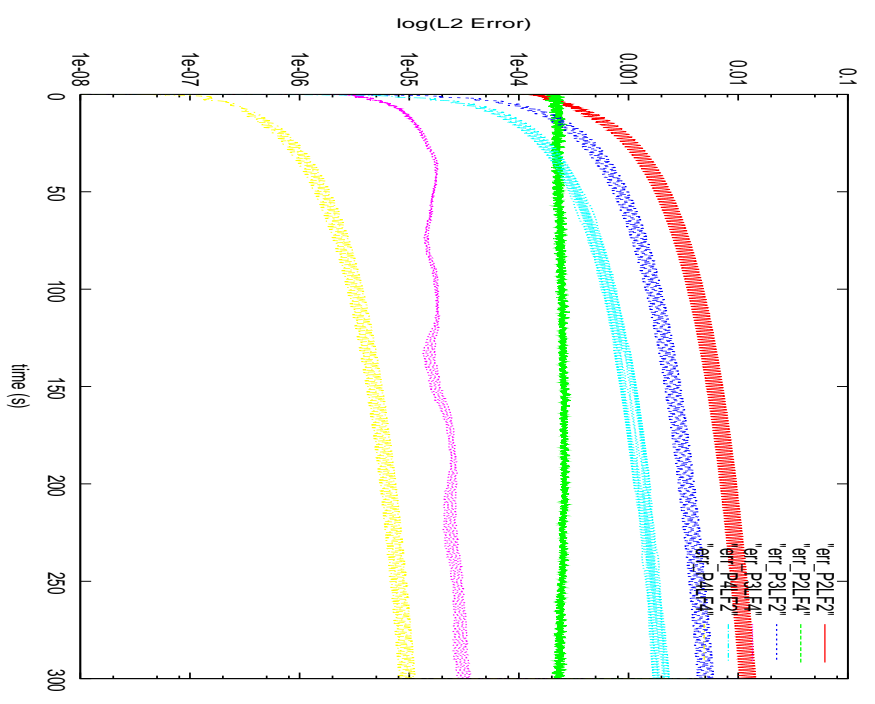

Figure 10. Evolution of the $L^{2}$ error of the solution of different schemes on 100 periods

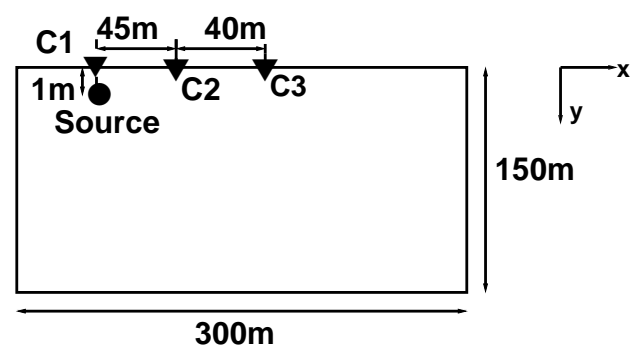

Figure 11. Geometry and position of the transducers for the Garvin experience

[10] D. Komatitsch And J.-P. Vilotte, “The spectral-element method: an efficient tool to simulate the seismic response of 2D and 3D geological structures", Bull. Seism. Soc. Am., vol. $88,368-392,1998$.

[11] K. MARfurt, "Accuracy of finite-difference and finite-element modeling of the scalar and elastic wave equations“, Geophysics, vol. 49 (5), 533-549, (1984).

[12] P. Moczo, J. Kristek, V. Vavrycuk, R.J. Archuleta and L. Halada, "3D heterogeneous staggered-grid finite-difference modeling of seismic with volume harmonic and arithmetic averaging of elastic moduli and densities", Bull. Seism. Soc. Am., vol. 92, 3042-3066, 2002.

[13] R. Mullen, T. Belytschko, "Dispersion analysis of finite element semidiscretizations of the two-dimensional wave equation“, Inter. J. Numer. Meth. Engrg., vol. 18 (1), 11-29, 1982.

[14] R. S. Phillips AND L. SARASON, "Singular symmetric positive fist order differential operators“, J. Math. Mech. , vol. 15, p. 235-272, 1966

[15] W. REED AND T. HILl, "Triangular mesh method for neutron transport equation", Tech. Rep. LA-UR-73-479, Los Alamos Scientific Laboratory, 1973. 
114 A R I M A - Volume $17-2014$
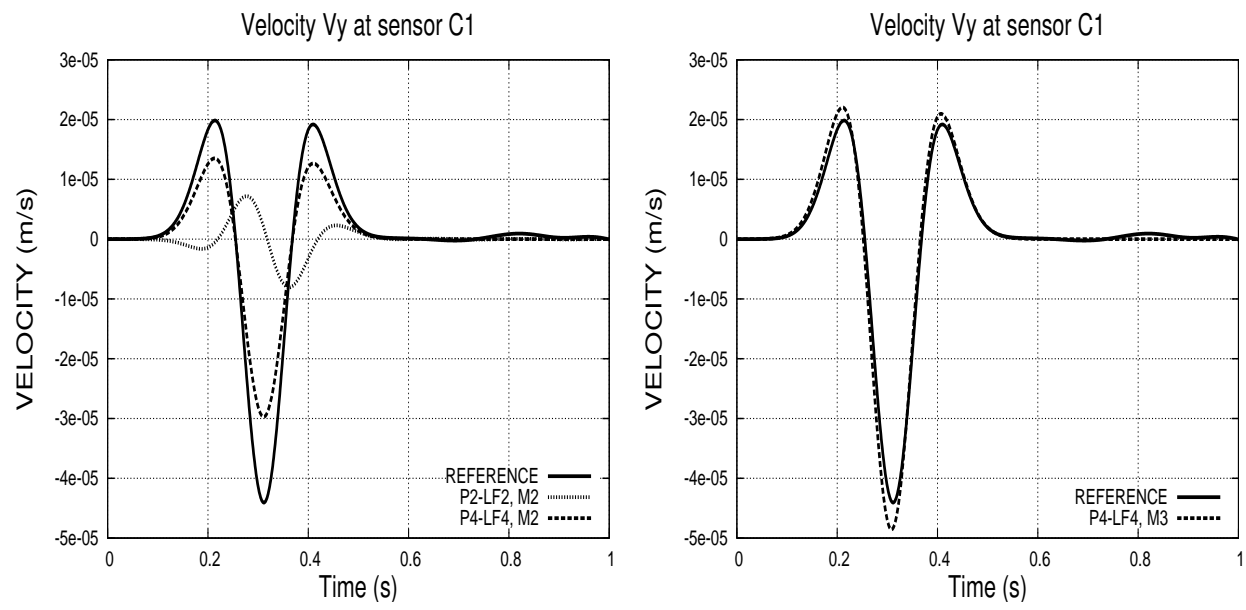

Figure 12. Velocities of the computed and the analytical solutions of the Garvin problem taken at the $\mathrm{C} 1$ transducer (Pk means DGK)

[16] F. J. SAnchez-Sesma, U. Iturraran-Viveros, “The classic Garvin's problem revisited", Bulletin of the Seismological society of America, vol. 96, no 4A, pp. 1344-1351, 2006

[17] H. Spachmann, R. Schuhmann, and T. Weilland, "High order explicit time integration scheme for Maxwell-s equations", Int. J. Numer. Model., vol. 15, 2002.

[18] J. VIRIEUX, "P-SV wave propagation in heterogeneous media: Velocity-stress finite difference method", Geophysics, vol. 51, 1986.

[19] J. L. YounG, "High order leapfrog methodology for the temporally dependent Maxwell's equations", Radio Science, num. 36, 2001. 

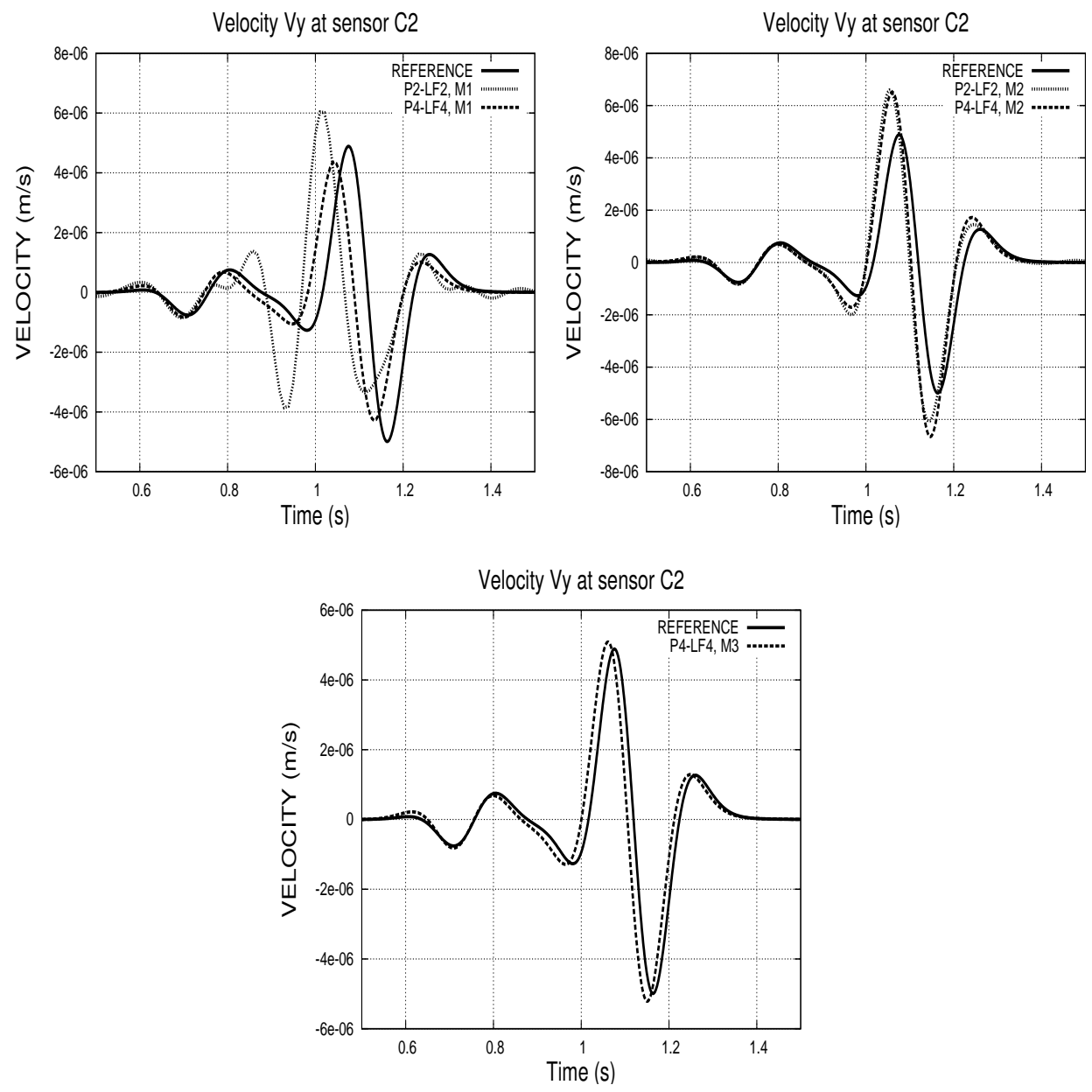

Figure 13. Velocities of the computed and the analytical solutions of the Garvin problem taken at the C2 transducer (Pk means DGk) 
116 A R I M A - Volume $17-2014$
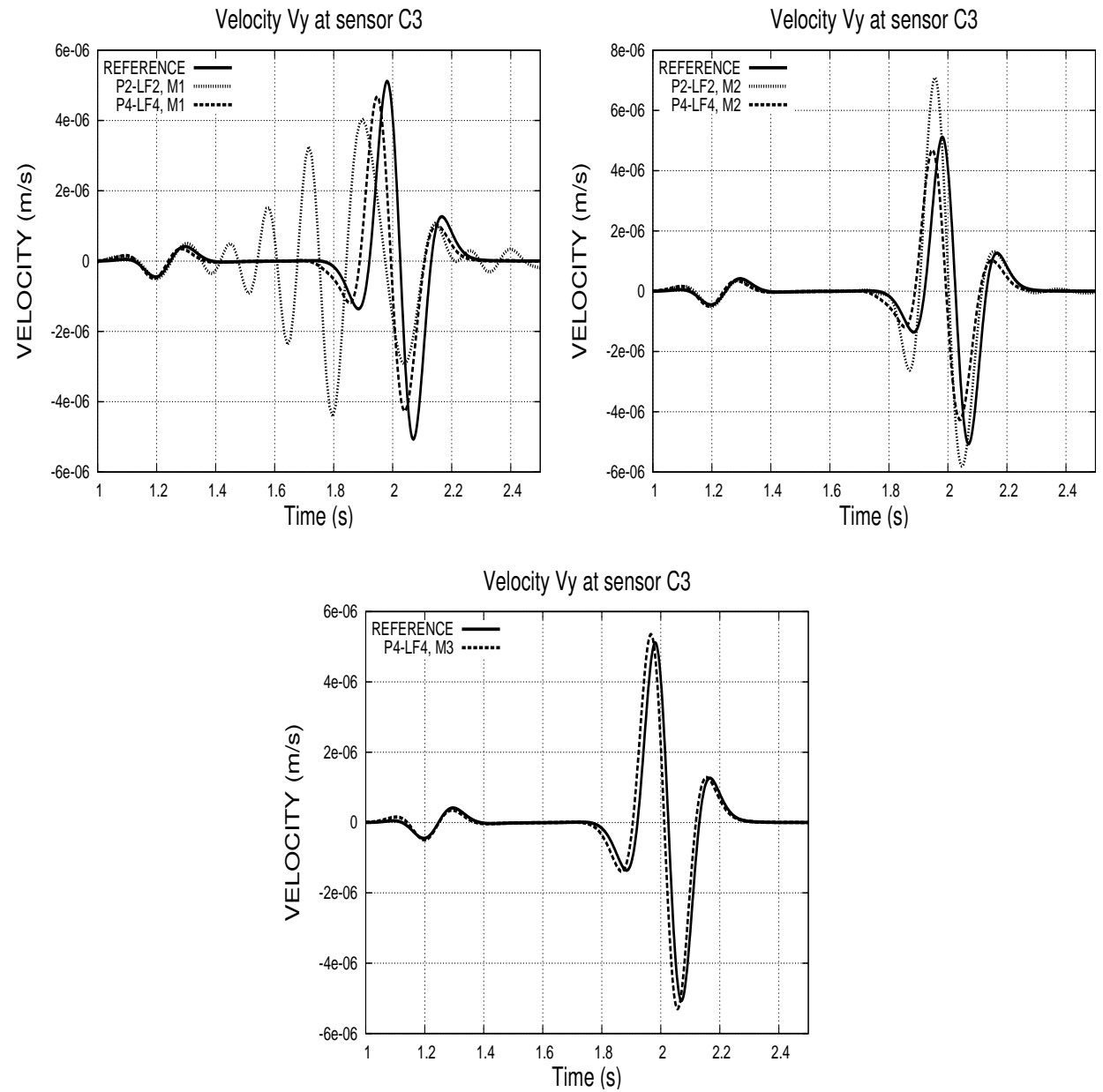

Figure 14. Velocities of the computed and the analytical solutions of the Garvin problem taken at the C3 transducer (Pk means DGk) 

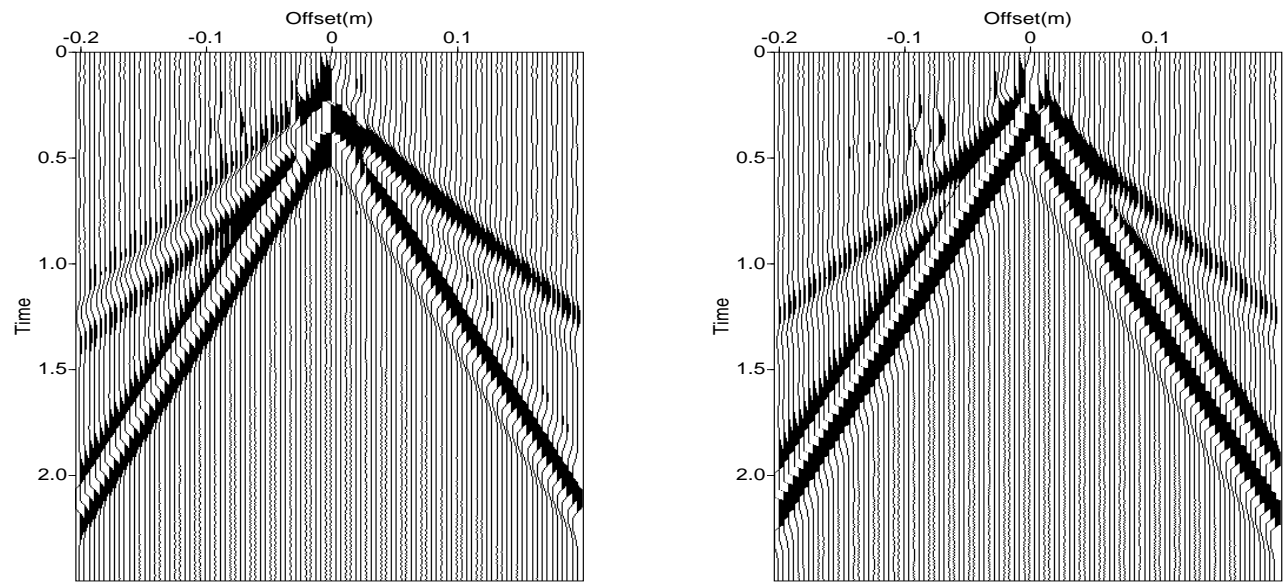

Figure 15. Sismograms of the horizontal and the vertical velocities of the computed solution of the Garvin problem 